\title{
dModifying the phenyl group of PUGNAc: Reactivity tuning to deliver selective inhibitors for $\boldsymbol{N}$-acetyl-D-glucosaminidases $\$$
}

\author{
Mitchell Hattie, ${ }^{a}$ Nevena Cekic, ${ }^{\mathrm{b}}$ Aleksandra, W. Debowski, ${ }^{\text {a,c }}$ David J Vocadlo, ${ }^{\text {b,d }}$ and Keith A. Stubbs ${ }^{\mathrm{a}^{*}}$ \\ Received (in $X X X, X X X) X$ th $X X X X X X X X X 20 X X$, Accepted $X$ th $X X X X X X X X X 20 X X$ \\ ${ }_{5}$ DOI: 10.1039/b000000x
}

The synthesis of analogues of the potent $\mathrm{N}$ acetylhexosamindase inhibitor PUGNAc are described. These compounds were assayed against a set of biologically important $\mathrm{N}$-acetyl-D-glucosaminidases and were found to 10 vary in both potency and selectivity.

Carbohydrate-processing enzymes are an important class of proteins found in nature involved in an array of biological processes including cell signalling, inflammation and protein quality control. ${ }^{1}$ Inhibitors are widely useful in helping to 15 understand this class of enzymes and especially the glycoside hydrolases (glycosidases). ${ }^{2-5}$ These inhibitors, including both natural products and unprecedented synthetic compounds enable researchers to understand catalysis by glycosidases, the tertiary structures of these enzymes, and their functional roles 20 in biological systems. Indeed these compounds have also formed the basis for the development of therapeutics. ${ }^{6-10}$

One sub-class of glycosidases of significant interest are the exo- $\mathrm{N}$-acetyl-D-glucosaminidases which process $\mathrm{N}$ acetylglucosamine residues from glycoconjugates. These 25 enzymes are ubiquitous in all domains of life where, for example, they play roles in peptidoglycan recycling, ${ }^{11}$ bacterial virulence $^{12}$ and competition ${ }^{13}$, as well as diverse roles in eukaryotes. ${ }^{14}$ As a result of the biological importance of these enzymes various different classes of inhibitor have 30 been investigated.

One set of compounds which stand out as useful inhibitors of glycosidases are the hydroximolactones of which $\mathrm{O}$-(2acetamido-2-deoxy-D-glucopyranosylidene)amino $\mathrm{N}$ phenylcarbamate (PUGNAc, Figure 1A) is the best known 35 member. ${ }^{15,16}$ This compound is a potent inhibitor of exo- $\mathrm{N}$ acetyl-D-glucosaminidases. ${ }^{15,17-21}$ The structural basis for the potency of hydroximolactones including PUGNAc lies, in part from these compounds possessing an $s p^{2}$-hybridised carbon at $\mathrm{C}-1$ which is thought to constrain these compounds to adopt a 40 conformation resembling the geometry of the dissociative $\mathrm{SN}_{2}$-like transition state of the reaction catalysed by glycosidases (Figure 1B). Another important feature is the oxime substituent, which mimics the aglycon of a natural substrate to harness additional binding energy. ${ }^{15}$

45 Despite PUGNAc having the benefits of high potency and membrane permeability, which make it suitable for cell-based studies, it lacks the selectivity, ${ }^{17}$ which is critically important especially in eukaryotic systems containing multiplie exo- $\mathrm{N}$ acetyl-D-glucosaminidases. This selectivity problem is also 50 shared by other glycosidase inhibitors, including various azaand iminosugars. ${ }^{22,23}$ In an effort to address the problems associated with selectivity, inhibitors have been designed where the compound scaffold is retained but elaborated with various chemical moieties. This method has met with success ${ }_{55}$ for various glycosidase inhibitors. ${ }^{17,24-27}$

Some efforts have been made to improve the selectivity of PUGNAc toward specific exo- $N$-acetyl-D-glucosaminidases. For example, the C-4 epimer of PUGNAc, termed GalPUGNAc, has been shown to be selective for the lysosomal ${ }_{60}$ exo- $N$-acetyl-D-glucosaminidases ${ }^{28}$ and the $N$-acyl group of PUGNAc has been modified to obtain modest selectivity for a nucleocytoplasmic exo- $N$-acetyl-D-glucosaminidase found in multicellular eukaryotes termed $O$-GlcNAcase (OGA) (Figure 1C). ${ }^{29,30}$

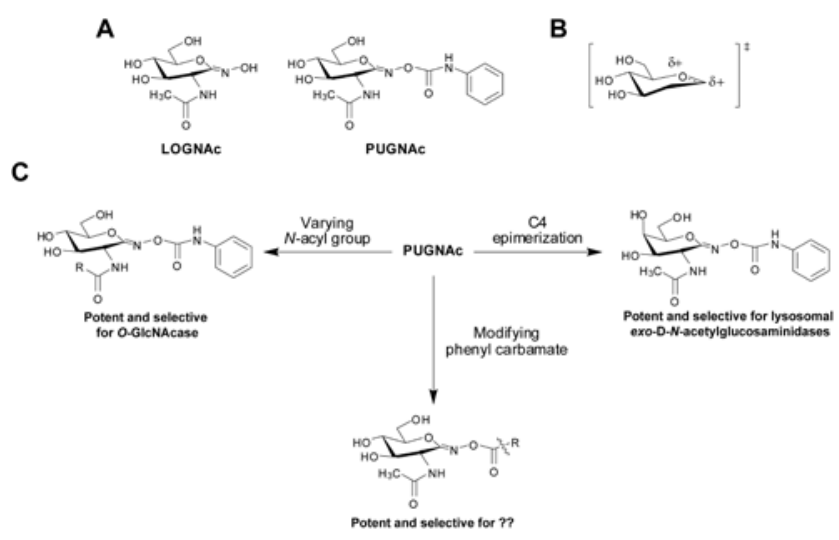

65 Figure 1. (A) The chemical structure of PUGNAc and hydroximolactone LOGNAc. (B) The carbohydrate portion of the putative transition state of exo- $N$-acetyl-D-glucosaminidases. (C) Modifications of PUGNAc to induce selectivity.

One part of the chemical structure of PUGNAc which has 70 not received much attention in terms of developing potent and selective inhibitors for exo- $N$-acetyl-D-glucosaminidases, is modification of the phenyl moiety (Figure 1C). This is despite this feature being important to binding as manifested by the markedly tighter binding of PUGNAc than 2-acetamido-275 deoxy-D-gluconhydroximo-1,5-lactone (LOGNAc), to various 
exo- $N$-acetyl-D-glucosaminidases. ${ }^{15,19}$ Also relevant is that exo- $N$-acetyl-D-glucosaminidases have different substrate specificites ${ }^{31-33}$ which is reflected in different active site architectures in the region bound by the phenylcarbamate group. ${ }^{18,20,34,35}$ Based on these considerations we felt that modification of the phenylcarbamate group would yield new inhibitors of exo- $N$-acetyl-D-glucosaminidases that might possess selectivity for one enzyme over another. Here we present a divergent synthesis for preparing analogues of 10 PUGNAc where the carbamoyl moiety is varied in terms of size, shape and hydrophobicity. Using enzymatic assays, with well characterized human exo- $N$-acetyl-D-glucosaminidases, we evaluate these molecules for potency and selectivity.

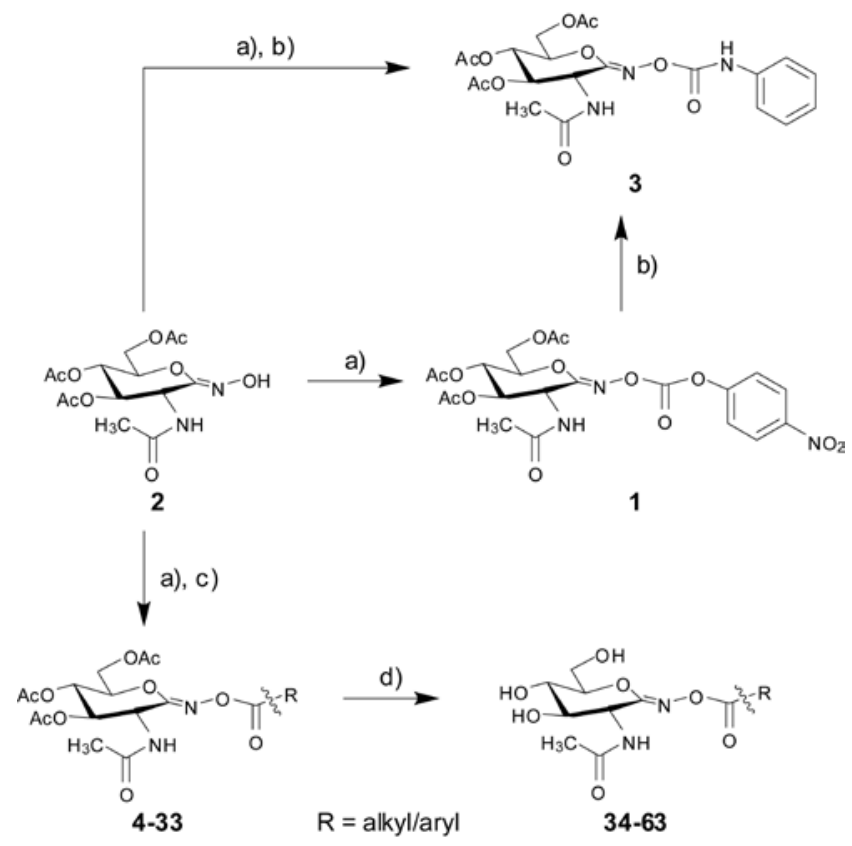

15

Scheme 1. a) 4-nitrophenyl chloroformate, DIPEA, THF. b) Aniline, DIPEA. c) Amine or aniline-based compound, DIPEA. d) $\mathrm{NH}_{3}, \mathrm{MeOH}$.

To realise a divergent synthetic pathway we hypothesized that the colourigenic carbonate $\mathbf{1}$ would serve as a useful 20 intermediate because formation of the carbamate can be indirectly monitored by observation of the liberated 4nitrophenolate anion. Thus, treatment of the readily prepared hydroximolactone 2, which is prepared using either the $\mathrm{N}$ acetyl $^{16,36}$ or Boc-protected ${ }^{29}$ synthetic methodologies, with 425 nitrophenyl chloroformate gave the desired carbonate $\mathbf{1}$ in good yield (Scheme 1). To test whether 1 would be suitably reactive with either substituted anilines or amines, we reacted 1 with aniline and, quite gratifyingly, obtained the phenyl carbamate $\mathbf{3}^{36}$ in good yield. To further improve the efficiency 30 of the method we felt that the reaction to give the carbamate 3 from the carbonate 2 could work in a one-pot strategy. Thus treatment of $\mathbf{2}$ to give the presumed carbonate in situ followed by addition of aniline gave the carbamate $\mathbf{3}$ in good overall yield (Scheme 1).

35 Using this one-pot methodology we treated the hydroximolactone $\mathbf{2}$ with a variety of aniline and amine derivatives that were comprised of a broad spectrum of materials with different size, shape and hydrophobic/hydrophilic propeties to yield a suite of 40 acetylated analogues 4-33 (Scheme 1). Reaction times to prepare the carbamates were variable, and were mainly dependent on the nucleophilicity of the amine utilised. Of interest is that the yields of the reactions were not generally affected by the nucleophilicity of the amine. Generally, the 45 primary amines reacted completely with the in situ formed carbonate 1, as judged by TLC, within one hour, however anilines required up to four days for complete reaction at room temperature. Removal of the $O$-acetyl protecting groups was achieved using ammonia in methanol to give the desired 50 triols 34-63 in good overall yield.

With these compounds in hand we evaluated them as potential inhibitors of three well characterised human enzymes, $\beta$-hexosaminidase B (HexB), NAGLU and OGA. These three enzymes, despite all being exo- $N$-acetyl-D55 glucosaminidases, act on different substrates and their loss of function within cells give rise to distinct celluar phenotypes. For HexB and NAGLU, which are both lysosomal enzymes, their respective natural susbtrates are glycosphingolipids, bearing either $\mathrm{N}$-acetylglucosamine or $\mathrm{N}$ 60 acetylgalactosamine, ${ }^{31}$ and heparan sulfate. ${ }^{33}$ Dysfunction of either of these enzymes leads to the lysosomal storage disorders Tay-Sachs and Sandhoff diseases ${ }^{37}$ for HexB and Mucopolysaccharidosis IIIB for NAGLU. ${ }^{38}$ The enzyme OGA acts on different substrates by removing $N$-acetylglucosamine 65 residues from post-translationally modified nucleocytoplasmic glycoproteins $^{32}$ and dysfunction of this enzyme has been linked to a variety of diseases. ${ }^{39}$ PUGNAc inhibits these enzymes ${ }^{15,18,40}$ with respective $K_{\mathrm{I}}$ values of $36 \mathrm{nM}^{17}, 7.1 \pm 0.3$ $\mu \mathrm{M}$ and $46 \mathrm{nM}^{17}$. Using an appropriate substrate (MU- $\beta$-D${ }_{70}$ GlcNAc for HexB and OGA and MU- $\alpha-D-G l c N A c$ for NAGLU, see Supporting Information) we screened compounds 34-63 at two concentrations (100 nM and $10 \mu \mathrm{M}$ ) to gain a sense of the overall potency and selectivity of the compounds. We observed that these compounds are all 75 inhibitors of these enzymes to varying extents (Figure 2).

Analysis of the phenyl derivatives reveals 34-37, unsurprisingly, that this group of analogues generally shows similar potency to that of PUGNAc. Of note is the benzyl derivative 37, even though the molecule has increased rotation 80 of the phenyl ring due to the methylene spacer, the potency against the enzymes was not severely affected. The electronics of the phenyl ring seems to be important for inhibiton for all the enzymes tested with the electron donating methoxy group on 35 reducing the potency of the scaffold relative to ${ }_{85}$ PUGNAc, whilst electron deficient $\mathbf{3 6}$ showed similar potency for HexB and OGA relative to PUGNAc. A potential reason for this observation lies in the binding interactions the phenyl ring makes in the +1 subsites of OGA and HexB. ${ }^{35,41}$

Generally the potency of the cyclic aliphatic derivatives 38${ }_{90} \mathbf{4 2}$, decreased with increasing ring size with the exception of the $N$-cyclohexyl derivative $\mathbf{3 8}$, as it was more potent for HexB and OGA than the $N$-cyclopentyl derivative $\mathbf{4 0}$. Interestingly, both $\mathbf{3 8}$ and $\mathbf{4 0}$ but not the cyclobutyl derivative 39 demonstrated selectivity for HexB over OGA. The 95 percentage inhibition of the amino acid derived analogues 

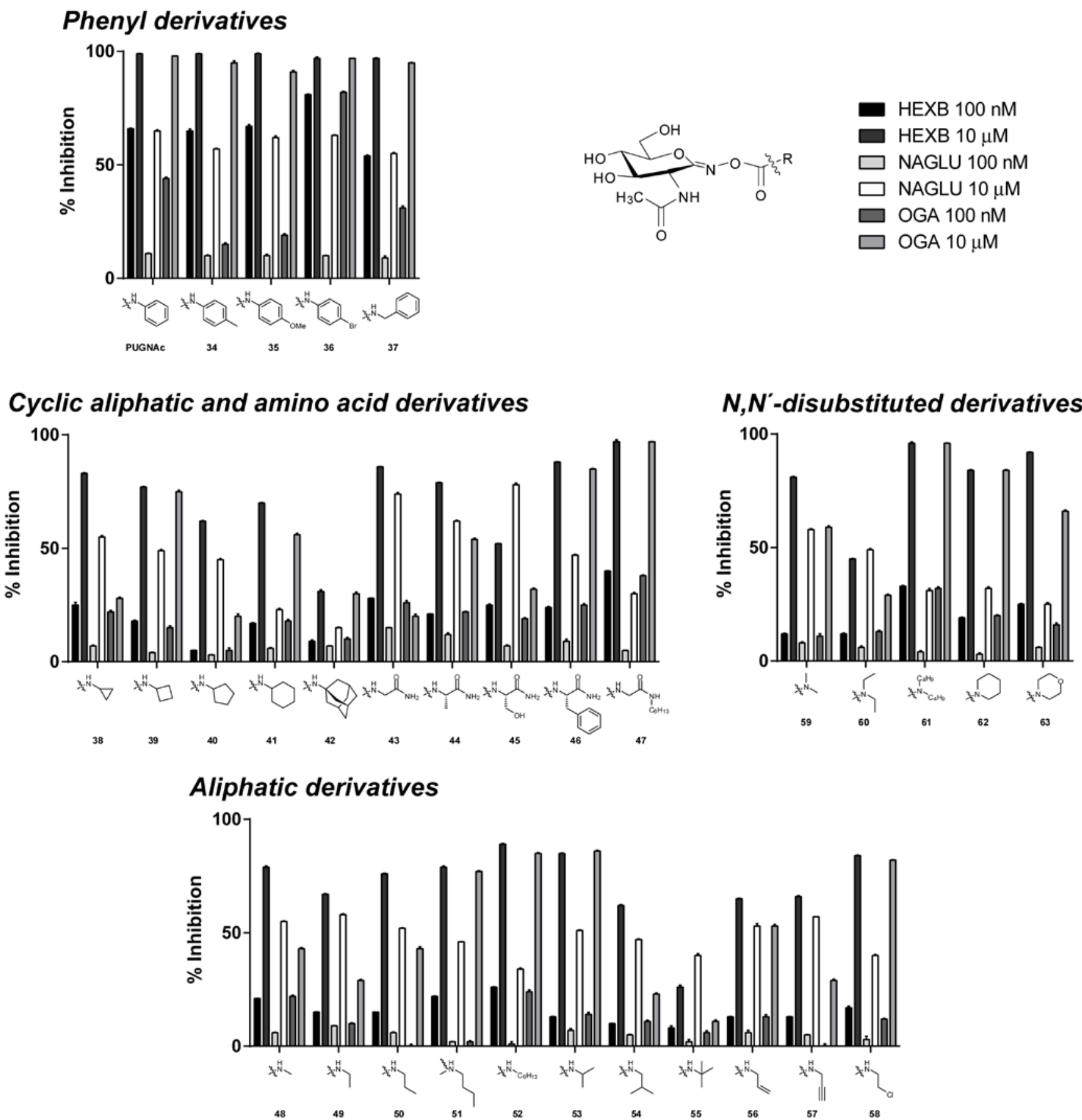

Figure 2. Percentage inhibition assays of the suite of PUGNAc-like compounds against three well characterized human enzymes HexB, NAGLU and OGA, all of which have been demonstrated to be inhibited by PUGNAc, using the substrates MU- $\beta$-D-GlcNAc for HexB and OGA and MU- $\alpha$-D-GlcNAc for NAGLU. All assays were carried out in triplicate with the error representing the standard error in the average of the measurements.

5 43-47 demonstrated that the glycine derivative 43 was selective for HexB over OGA. Notably, the serine derivative 45 showed completely reversed selectivity as compared to PUGNAc and was selective for NAGLU over the the other two enzymes. This may be due to the polar serine group 10 mimicking elements of the natural substrate of NAGLU which is the polar molecule heparan sulfate. The additional hexyl chain of 47 proved to increase the potency of the parent scaffold $\mathbf{4 3}$ for HexB and particularly OGA, suggesting that these enzymes require some degree of hydrophobic character 15 in the +1 subsite. However, this substituent was deleterious for inhibition of NAGLU.

The aliphatic derivatives $\mathbf{4 8 - 5 8}$ were all less potent inhibitors than PUGNAc against the three $\mathrm{N}$ acetylhexosaminidases. The $N$-2-chloroethyl derivative $\mathbf{5 8}$ did 20 not show significant differences compared to the $N$-ethyl and $N$-propyl analogues 49 and 50, which may be of interest in terms of enabling further functionalisation. 
The $N$-isobutyl derivative $\mathbf{5 4}$ showed selectivity for HexB over OGA and the unsaturated derivatives 56 and 57 also showed similar selectivity when compared to $\mathbf{5 0}$. The compounds were not as potent as expected despite the extra 5 electron density located in the aglycone portion of these molecules. The alkyne 57 was selective for HexB over OGA and so sets the stage for this molecule potentially being used as a synthon in click chemistry based library approaches to generate triazole-based inhibitors of exo- $\mathrm{N}$-acetyl-D10 glucosaminidases. The $N, N$-disubstituted derivatives 59-63, which lack one less hydrogen bond donor, were prepared to investigate the role that this H-bond may play in potency and selectivity. These compounds were inhibitors of all the enzymes tested, with 59, 60 and 63 showing selectivity for 15 HexB over OGA.

With these activity assays in hand several inhibitors were carried forward to the next stage of evaluation, where $K_{\mathrm{I}}$ values were obtained against the enzymes (Table 1 ). The trends observed in the rapid screen assay were consistent with 20 the $K_{\mathrm{I}}$ values obtained. Inhibitors lacking the phenyl ring were significantly less potent than PUGNAc. The assay results were consistent with $\mathbf{3 5}$ being a worse inhibitor

Table 1. Inhibition constants of inhibitors against HexB, NAGLU and OGA.

\begin{tabular}{cccc}
\hline & & $K_{\mathrm{I}}(\mu \mathrm{M})$ & \\
\hline Compound & HexB & NAGLU & OGA \\
PUGNAc & $0.036^{17}$ & $7.1 \pm 0.3$ & $0.046^{17}$ \\
$\mathbf{3 4}$ & $0.021 \pm 0.005$ & $39 \pm 3.4$ & $0.028 \pm 0.007$ \\
$\mathbf{3 5}$ & $0.111 \pm 0.019$ & $19 \pm 2.1$ & $0.178 \pm 0.042$ \\
$\mathbf{3 6}$ & $0.047 \pm 0.002$ & $21 \pm 4.3$ & $0.056 \pm 0.016$ \\
$\mathbf{4 0}$ & $14 \pm 3.5$ & $122 \pm 19$ & $230 \pm 78$ \\
$\mathbf{4 3}$ & $1.3 \pm 0.17$ & $8.6 \pm 0.9$ & $78 \pm 26$ \\
$\mathbf{4 5}$ & $45 \pm 16$ & $4.3 \pm 1.4$ & $420 \pm 140$ \\
$\mathbf{5 4}$ & $17 \pm 4$ & $105 \pm 15$ & $220 \pm 75$ \\
$\mathbf{5 5}$ & $161 \pm 8$ & $195 \pm 19$ & $188 \pm 72$ \\
$\mathbf{6 3}$ & $0.77 \pm 0.031$ & $222 \pm 28$ & $11 \pm 4.8$ \\
\hline
\end{tabular}

25

compared to PUGNAc while $\mathbf{3 4}$ and $\mathbf{3 6}$ showed similar potency for HexB and OGA relative to PUGNAc. Consistent with the assay results, good selectivity was observed for the amino acid analogues $\mathbf{4 3}$ and $\mathbf{4 5}$, with $\mathbf{4 3}$ having over 50-fold 30 selectivity for HexB and 10 -fold for NAGLU, against OGA. This presents potentially a useful scaffold for synthetic elaboration. The serine analogue $\mathbf{4 5}$ is potent against NAGLU, relative to PUGNAc, but is more selective for this enzyme against HexB compared to $\mathbf{4 3}$. As a result, compound $\mathbf{4 5}$ could 35 serve as an efficient starting point when preparing potential selective inhibitors for NAGLU. Interestingly the morpholine derivative 63 is $>10$-fold more selective for HexB over the other enzymes and the cyclopentyl derivative 40, whilst having lost potency compared to PUGNAc, is $\sim 10$-fold 40 selective for HexB over the other enzymes.

In conclusion we have developed a divergent synthetic route that enables the rapid synthesis of a series of PUGNAclike inhibitors bearing different carbamate groups. Using assays that allow for rapid screening we demonstrated that all 45 the compounds prepared were inhibitors against the exo- $N$ acetyl-D-glucosaminidases tested, HexB, NAGLU and OGA. Difference in potencies is consistent with differences in the active site architectures between these enzymes and suggests that modification of PUGNAc at this position warrants further 50 investigation. Indeed these further investigations, using the strategy outlined here may reveal even more selective inhibitors for these enzymes. Finally, we anticipate that these observations will, in combination with other structural variations, lead to highly selective tool compounds for these 55 enzymes that could be used in cells and tissues.

The authors wish to thank the Centre for Microscopy, Characterisation and Analysis, The University of Western Australia, which are supported by University, State and Federal Government funding. KAS also thanks the Australian 60 Research Council for funding. $\mathrm{MH}$ thanks the University of Western Australia for an Australian Postgraduate Award and a Jean Rogerson Postgraduate Scholarship. AWD thanks the National Health and Medical Research Council for funding (APP1073250). DJV thanks the Canadian Institute of Health ${ }_{65}$ Research (MOP-123341) and Brain Canada for support of this research. DJV also thanks the Canada Research Chairs Program for support as a Tier I Canada Research Chair in Chemical Glycobiology.

\section{Notes and references}

$70{ }^{a}$ School of Chemistry and Biochemistry, The University of Western Australia, Crawley, WA 6009, Australia. E-mail: keith.stubbs@uwa.edu.au

${ }^{b}$ Department of Chemistry, Simon Fraser University, Burnaby, British Columbia, V5A 1S6, Canada

$75{ }^{c}$ School of Pathology and Laboratory Medicine, The University of Western Australia, Crawley, WA 6009, Australia

${ }^{d}$ Department of Molecular Biology and Biochemistry, Simon Fraser University, Burnaby, British Columbia, V5A 1S6, Canada

†Dedicated to the memory of Associate Professor Emilio Ghisalberti.

80 ‡Electronic Supplementary Information (ESI) available: See DOI: $10.1039 / \mathrm{b} 000000 \mathrm{x} /$

1.Essentials of Glycobiology, Cold Spring Harbor Laboratory Press, Cold Spring Harbor, New York, 2009.

85 2. N. Asano, Cell. Mol. Life Sci., 2009, 66, 1479-1492.

3. T. M. Gloster and G. J. Davies, Org. Biomol. Chem., 2010, 8, 305-320.

4. T. M. Gloster and D. J. Vocadlo, Nat. Chem. Biol. , 2012, 18, 683-694.

5. L. I. Willems, J. Jiang, K. Y. Li, M. D. Witte, W. W. Kallemeijn, T. J. Beenakker, S. P. Schroder, J. M. Aerts, G. A. van der Marel, J. D. Codee and H. S. Overkleeft, Chem. Eur. J., 2014, 20, 10864-10872.

6.D. D. Schmidt, W. Frommer, B. Junge, L. Muller, W. Wingender, E. Truscheit and D. Schafer, Naturwissenschaften, 1977, 64, 535-536.

7. S. Horii, H. Fukase, T. Matsuo, Y. Kameda, N. Asano and K. Matsui, J. Med. Chem, 1986, 29, 1038-1046.

95 8. M. von Itzstein, W. Y. Wu, G. B. Kok, M. S. Pegg, J. C. Dyason, B. Jin, T. Van Phan, M. L. Smythe, H. F. White, S. W. Oliver and et al., Nature, 1993, 363, 418-423.

9. A. R. Sawkar, W. C. Cheng, E. Beutler, C. H. Wong, W. E. Balch and J. W. Kelly, Proc. Natl. Acad. Sci. USA, 2002, 99, 15428-15433.

100 10. S. A. Yuzwa, M. S. Macauley, J. E. Heinonen, X. Shan, R. J. Dennis, Y. He, G. E. Whitworth, K. A. Stubbs, E. J. McEachern, G. J. Davies and D. J. Vocadlo, Nat. Chem. Biol., 2008, 4, 483-490.

11. Q. Cheng, H. Li, K. Merdek and J. T. Park, J. Bacteriol., 2000, 182, 4836-4840.

105 12. S. J. King, K. R. Hippe and J. N. Weiser, Mol. Microbiol., 2006, 59, 961-974. 
13. J. Wada, T. Ando, M. Kiyohara, H. Ashida, M. Kitaoka, M. Yamaguchi, H. Kumagai, T. Katayama and K. Yamamoto, Appl. Environ. Microbiol., 2008, 74, 3996-4004.

14. M. S. Macauley and D. J. Vocadlo, Biochim. Biophys. Acta. , 2010, 1800, 107-121.

15. M. Horsch, L. Hoesch, A. Vasella and D. M. Rast, Eur. J. Biochem., 1991, 197, 815-818.

16. D. Beer, J. L. Maloisel, D. M. Rast and A. Vasella, Helv. Chim. Acta, 1990, 73, 1918-1922.

10 17. M. S. Macauley, G. E. Whitworth, A. W. Debowski, D. Chin and D. J. Vocadlo, J. Biol. Chem., 2005, 280, 25313-25322.

18. E. Ficko-Blean, K. A. Stubbs, O. Nemirovsky, D. J. Vocadlo and A. B. Boraston, Proc. Natl. Acad. Sci. USA 2008, 105, 6560-6565.

19. K. A. Stubbs, M. Balcewich, B. L. Mark and D. J. Vocadlo, J. Biol. 15 Chem., 2007, 282, 21382-21391.

20. T. Sumida, K. A. Stubbs, M. Ito and S. Yokoyama, Org. Biomol. Chem., 2012, 10, 2607-2612.

21. M. Hattie, T. Ito, A. W. Debowski, T. Arakawa, T. Katayama, K. Yamamoto, S. Fushinobu and K. A. Stubbs, Chem. Commun., 2015,

20 51, 15008-15011.

22. Iminosugars: From synthesis to therapeutic applications, John Wiley \& Sons, Ltd., Chichester, 2007.

23. G. Horne, F. X. Wilson, J. Tinsley, D. H. Williams and R. Storer, Drug Discov. Today, 2011, 16, 107-118.

25 24. U. Andersson, T. D. Butters, R. A. Dwek and F. M. Platt, Biochem. Pharmacol. , 2000, 59, 821-829.

25. R. J. van den Berg, W. Donker-Koopman, J. H. van Boom, H. M. Aerts and D. Noort, Bioorg Med Chem, 2004, 12, 891-902.

26. P. Liang, W. Cheng, Y. Lee, H. Yu, Y. Wu, Y. Lin and C. Wong, ChemBioChem, 2006, 7, 165-173.

27. H. C. Dorfmueller, V. S. Borodkin, M. Schimpl, X. Zheng, R. Kime, K. D. Read and D. M. van Aalten, Chem. Biol., 2010, 17, 1250-1255.

28. K. A. Stubbs, M. S. Macauley and D. J. Vocadlo, Angew. Chem. Int. Ed., 2009, 48, 1300-1303.

35 29. K. A. Stubbs, N. Zhang and D. J. Vocadlo, Org. Biomol. Chem., 2006, 4, 839-845.

30. E. J. Kim, M. Perreira, C. J. Thomas and J. A. Hanover, J. Am. Chem. Soc., 2006, 128, 4234-4235.

31. E. Klenk, Z. Physiol. Chem., 1942, 273, 76.

40 32. C. R. Torres and G. W. Hart, J Biol Chem, 1984, 259, 3308-3317.

33. E. Neufeld and J. Muenzer, eds. C. Sciver, A. Beaudet, W. Sly and D. Valle, McGraw-Hill, New York, 1995, pp. 2465-2494

34. R. J. Dennis, E. J. Taylor, M. S. Macauley, K. A. Stubbs, J. P. Turkenburg, S. J. Hart, G. N. Black, D. J. Vocadlo and G. J. Davies,

$45 \quad$ Nat. Struct. Mol. Biol., 2006, 13, 365-371.

35. M. Balcewich, K. A. Stubbs, Y. He, T. James, G. J. Davies, D. J. Vocadlo and B. L. Mark, Protein Sci., 2009, 18, 1541-1551.

36. D. Beer and A. Vasella, Helv. Chim. Acta, 1985, 68, 2254-2274.

37. B. Triggs-Raine, D. J. Mahuran and R. A. Gravel, Adv. Genet., 2001,

$50 \quad$ 44, 199-224.

38. G. Yogalingam and J. J. Hopwood, Hum. Mutat., 2001, 18, 264-281.

39. G. W. Hart, M. P. Housley and C. Slawson, Nature, 2007, 446, 10171022.

40. D. L. Dong and G. W. Hart, J. Biol. Chem., 1994, 269, 19321-19330.

55 41. M. S. Macauley, A. K. Bubb, C. Martinez-Fleites, G. J. Davies and D. J. Vocadlo, J. Biol. Chem., 2008, 283, 34687-34695. 